\title{
Deliberate Ambiguity in a Finite Environment: The Urban Ecology of Artificial Items
}

\author{
ABRAHAM AKKERMAN* \\ Department of Geography, University of Saskatchewan, Saskatoon, SK S7N 0W0, Canada
}

(Received 30 May 1999)

\begin{abstract}
A distinction is made between visual declaration and virtual usage of artificial items within a physical environment, such as a street. Visual declaration is a formal pictorial designation, or a function, e.g. "decoration," of an item, such as a "planter." Virtual usage refers to the item when it is used in lieu of another item. The formal designation, "sitting," customarily designated to an item such as "bench," could also be a virtual usage of the item "planter." The question asked is, "What is the relationship between items, given their formal, visual declaration and their informal, virtual, usage?" An artificial item, according to its visual declaration, is referred to as a 'visual' or 'real item'. Each visual item has the property of being used as another item by virtue of its undeclared usage. Pending on the item's design and configuration, a visual item can be then substituted for another visual item. An artificial item, thus, attains deliberate ambiguity between its formal designation and its virtual usage. This ambiguity between visual declaration and virtual usage can be quantified. Within the full domain of $\boldsymbol{n}$ possible usages, this relationship can be conveniently presented in a nonnegative matrix. It is shown that the inverse of this matrix belongs to a class of well-known matrices. This being the case, the relationship between visual and virtual properties of items within the environment can be formalized. The formalization throws further light on the emerging opportunities in streetscape design.
\end{abstract}

Keywords: Built environment, Street furniture, Virtual usage, Ambiguity

\section{INTRODUCTION}

We consider a set of artificial, physical items in a finite environment. The items under consideration are assumed to be visible. These items will be referred to as visual items (or simply as items), in contradistinction to items within the environment that may be hidden or unusable, and which are of no concern here. A pictorial designation of a visual item is defined as the item's declared, formal usage within the physical environment. We assume a semiotic, one-to-one correspondence between an item and its visual declaration. Each visual item, then, has one and only one visual declaration. Each item,

*Fax: +1 3069665680. E-mail: akkerman@sask.usask.ca. 
however, may have an additional, informal usage that corresponds to a formal pictorial designation of some other visual item. The possibility for an item to have a usage other than its formally declared usage, implies the potential for ambiguity, or vagueness, in the design and spatial configuration of usable objects (cf. Sainsbury, 1994). The attempt here will be to formulate a systematic recognition of deliberate ambiguity which often is in the core of applied public art. Such recognition is of significance to urban design, and to the design and configuration of street furniture in particular.

The orderly treatment of ambiguity and imprecision in urban design has been of emerging concern not only to architects (Venturi et al., 1972; Broadbent, 1990), but indirectly also to planners (Akkerman, 1992; Kim, 1992). The following is an attempt to formalize the notions of ambiguity and imprecision, applicable to artificial objects in a streetscape, without loss of generality for other finite physical environments. The question addressed here is, "What is the relationship between items, given their formal, visual declaration and their informal, virtual, usage?" In a particular example, a street environment may include "bench," "street sign," "railing," "bollard" and "planter". Each item has a formal pictorial declaration in which, for example, "bench" is related to "sitting," a "planter" is related to "plant decoration" and "street sign" is related to "orientation". Pending its own design, however, a planter could have additional possible usages as well; for example "sitting" and "orientation."

In a more specific context, assume that within the environment a distribution of desired items by pictorial declaration exists. Thus, in a streetscape, there may be a need for 3 items for "sitting," 2 items for "orientation," 7 items for "plant decoration," etc. Given the fact that visual items possess categories of usage other than their pictorial declarations, the question may be asked, "What is the minimum number of physical items in the streetscape, satisfying the above condition?" It will be shown in the following that the ambiguity relationship between the visual declaration of physical items, and the usage categories of items within a finite environment, can be presented as a formal numerical relation.

The formalized ambiguity relationship implies two methodological views unique to street and street-furniture design. The one is the observation of the streetscape as a stage-setting in a performing art, rather than as a static architectural construct (cf. Moughtin, 1992). The design, or redesign, of streetscape and street furniture must recognize, or deliberately invite, alternative uses of artificial items within the streetscape. The other view is the wilful choice of municipal hardware as an explicit streetscape feature. Both aspects of ambiguity in street design emerge within the context of postmodernist architecture. But significantly, both aspects seem to offer an opportunity to mold or redefine the characteristics and the volume of pedestrians in our streets.

\section{VISUAL AND VIRTUAL ITEMS}

Semiotic relationships within pedestrian space have led recently to attempts to quantify notions of access to, or use of such objects as department store entrances (Scott, 1993). Similarly, the approach developed here assumes an expert evaluation of visual items within a delineated, physical environment. All items are presumed to belong to one of $n$ distinct categories, each category being the visual declaration of an item. Consider thus a delineated physical environment within which a finite number of physical items is present. The distribution of physical items by the $n$ distinct categories is given by a row vector $\mathbf{k}$,

$$
\mathbf{k}=\left(k_{1}, \ldots, k_{i}, \ldots, k_{n}\right),
$$

$k_{i}$ denoting the number of physical items in category $i$, present in the environment.

The relationship between the categories of items is such that an item in a given category $i$ sometimes serves as a virtual item in some other category $j$; i.e., the item $i$ has virtual usage corresponding to item $j$ 
$(i, j=1, \ldots, n)$. Such relationship could, in fact, be specified by deliberate design of the item (for example, a planter deliberately designed to serve also as a bench) or by the spatial configuration of items within the environment (for example, a planter far away from a bench). In the context of public art, the design may entail specific intention by an artist and actual use (Pennartz, 1992). Here, the evaluation of the item's usage is assumed to be by actual observation or expert input. Thus, virtual usage corresponding to a category $j$ of an item in category $i$, could be specified by some observed frequency of usage, or availability, of item $i$ as item $j$. Such alternate usage or availability, therefore, also indicates a measure of deliberate ambiguity implicit in the item.

Let us denote a numeric measure of ambiguity for item $i$ to be a virtual item $j$, as $a_{i j}$. Let the ambiguity $a_{i j}$ of item $i$ as item $j$, be implicit in the item's design and spatial configuration, and let $a_{i j}$ be given by an expert as a nonnegative numerical value less than or equal to 1 . Since each physical item $i$ has a defined formal usage given by its visual declaration, let the value $a_{i i}$ be set to 1 as a convention. Any other number $a_{i j}$ will be then usually 0 or a fraction of 1 , denoting the relative usage of the physical item $i$ as a virtual item $j$.

The numbers $a_{i j}$ can be ordered in a square matrix A, referred to henceforth as the ambiguity matrix. Consider, then, the ambiguity matrix $\mathbf{A}$, each element $a_{i j}$ of which denotes the availability of item in category $i$ as a virtual item in category $j$ :

$$
\mathbf{A}=\left[\begin{array}{ccccc}
1 & a_{12} & a_{13} & \ldots & a_{1 n} \\
a_{21} & 1 & a_{23} & \ldots & a_{2 n} \\
& & & \ddots & \\
a_{n 1} & a_{n 2} & a_{n 3} & \ldots & 1
\end{array}\right]
$$

Let the sum of elements $a_{i j}$ in row $i$ be $s_{i}$. The numbers $s_{i}$ are of particular interest. A brief inspection shows that $s_{i}$ is the number of all virtual items available from one physical item of category $i$ within the environment, and that $s_{i}$ is equal to or greater than 1 . This means that for a desired distribution of virtual items in the environment, we will usually require a visual distribution that entails less items.

\section{AMBIGUITY AS A LINEAR PROBLEM}

Consider, in particular, the product $(\mathbf{k} \cdot \mathbf{A})$. A careful examination of $\mathbf{A}$ and $\mathbf{k}$ shows that $(\mathbf{k} \cdot \mathbf{A})$ is the distribution of virtual items within environment, as given by the distribution $\mathbf{k}$ of physical items. Let, then, $\mathbf{w}$ be a row vector of distribution of virtual items within the environment. From the above considerations it follows that

$$
\mathbf{k} \leq \mathbf{w}
$$

and

$$
\mathbf{k} \cdot \mathbf{A}=\mathbf{w} .
$$

Since both $\mathbf{k}$ and $\mathbf{w}$ must be nonnegative, the feasibility of Eq. (2) is limited to those vectors $\mathbf{w}$ for which,

$$
\mathbf{w} \cdot \mathbf{A}^{-1}=\mathbf{k} \geq \mathbf{0} .
$$

The restriction of Condition (3) applies to any distribution $\mathbf{w}$ of virtual items since the distribution vector $\mathbf{k}$ must not attain negative values in any of its entries.

As a means describing the formal relationship between the distributions of visual and virtual items within the environment, Condition (3) would be prohibitive for a generic nonnegative matrix $\mathbf{A}$. The matrix $\mathbf{A}$ in Eq. (2), however, has its diagonal elements 1 and its offdiagonal elements nonnegative, but smaller than 1. Matrices with their diagonal elements 1 are referred to as normalized matrices. Normalized matrices with small offdiagonal nonnegative elements, such as $\mathbf{A}$, are found in many contexts, notably in the Leontieff input-output model. It is often the case that a nonnegative square matrix such as A (having diagonal elements equal to or larger than 1 , and very small nonnegative offdiagonal elements), has an inverse, $\mathbf{A}^{-1}$, whose 
diagonal elements are all positive and whose offdiagonal elements are nonpositive, and equal or close to 0 . Matrices with all their diagonal elements equal to or larger than 1, and all their offdiagonal elements nonpositive and equal or very close to 0 , are referred to as nonsingular $\mathbf{M}$-matrices.

The identification of the matrix $\mathbf{A}^{-1}$ as a nonsingular M-matrix gives it a definite pattern. As a nonsingular M-matrix, $\mathbf{A}^{-1}$ can be expressed in the form

$$
\mathbf{A}^{-1}=s \mathbf{I}-\mathbf{D},
$$

where,

$$
\mathbf{D} \geq \mathbf{0}, \quad s \geq r(\mathbf{D})>\mathbf{0},
$$

with the following specifications: $s$ is a scalar, $\mathbf{I}$ is the identity matrix, $\mathbf{D}$ is a nonnegative nonsingular matrix, and $r(\mathbf{D})$ is the largest eigenvalue (known also as the Frobenius-Perron eigenvalue) of $\mathbf{D}$.

The significant finding of the theory on nonnegative matrices is that Eq. (4) ensures that for each nonnegative vector $\mathbf{k}$ there exists exactly one nonnegative vector $\mathbf{w}, \mathbf{k}=\mathbf{w} \cdot \mathbf{A}^{-1}$, as given by the matrix equation of Condition (3) (Berman and Plemmons, 1979).

\section{TARGET DISTRIBUTION OF VIRTUAL ITEMS}

Assume that $\mathbf{A}^{-1}$ is, indeed, an $\mathbf{M}$-matrix as in Eq. (4). If $\mathbf{A}^{-1}$ further acts also as a monotonous linear operator from $\mathbf{w}$ to $\mathbf{k}$, then there is a feasible range of distribution vectors $\mathbf{w}$ of virtual items, whose corresponding distribution vectors $\mathbf{k}$ of real items, are nonnegative.

The possibility of a feasibility range of distributions $\mathbf{w}$ of virtual items that correspond to distributions $\mathbf{k}$ of real items within the environment, has further implications. Of particular interest in street design is the minimum distribution $\mathbf{k}$ of physical items, such as street furniture, that achieves some desired overall usage. A desired usage can be expressed as a target distribution $\mathbf{w}$ of virtual items. From Inequality (1) it is clear that the physical distribution vector $\mathbf{k}$ will usually be less than the virtual distribution vector $\mathbf{w}$. From this perspective, the situation, $\mathbf{k}=\mathbf{w}$ (i.e., where $\mathbf{A}$ is the identity matrix), is the worst case for the relationship between real and virtual items. This relationship, given by Eq. (3) and represented by a nonnegative matrix $\mathbf{A}$, will be consistent with Inequality (1) if at least some offdiagonal elements of $\mathbf{A}$ are positive.

It is the virtual usage of items, such as street furniture, that ultimately should decide the inventory of items within a delimited space such as a streetscape. Table I shows a sample ambiguity relationship between 10 street-furniture categories (Gibbons and Oberholzer, 1991) representing both visual and virtual items. For simplicity, it is assumed that all items within a category, throughout the streetscape, are of identical design. For further simplicity we also assume that the ambiguity relationships represented by the numbers $a_{i j}$ do not

TABLE I Sample ambiguity, A, for selected street furniture

\begin{tabular}{rlllllllllll}
\hline & & 1 & 2 & 3 & 4 & 5 & 6 & 7 & 8 & 9 & 10 \\
\hline 1 & Bench & 1 & 0.1 & 0.1 & 0 & 0 & 0 & 0.1 & 0.2 & 0 & 0 \\
2 & Planter & 0.1 & 1 & 0.3 & 0 & 0 & 0.1 & 0.1 & 0.2 & 0 & 0.2 \\
3 & Litter bin & 0 & 0.1 & 1 & 0 & 0 & 0 & 0.2 & 0.1 & 0 & 0 \\
4 & Bollard & 0 & 0.1 & 0 & 1 & 0.1 & 0 & 0.3 & 0.4 & 0 & 0 \\
5 & Railing & 0.2 & 0 & 0.1 & 0.1 & 1 & 0 & 0.1 & 0.1 & 0 & 0 \\
6 & Gateway & 0 & 0 & 0 & 0 & 0.1 & 1 & 0.1 & 0.1 & 0 & 0 \\
7 & Lighting & 0.1 & 0.1 & 0 & 0.1 & 0 & 0.1 & 1 & 0.1 & 0.1 & 0 \\
8 & Signage & 0.1 & 0.1 & 0.1 & 0.1 & 0 & 0.1 & 0.1 & 1 & 0.1 & 0 \\
9 & Canopy & 0 & 0 & 0 & 0 & 0 & 0 & 0.1 & 0.1 & 1 & 0 \\
10 & Fountain & 0 & 0 & 0 & 0.1 & 0 & 0 & 0 & 0.2 & 0 & 1 \\
\hline
\end{tabular}


TABLE II Approximation of the inverse $\mathbf{A}^{-1}$, selected street furniture

\begin{tabular}{rrrrrrrrrr}
\hline 1.04 & -0.07 & -0.06 & 0.03 & -0.00 & 0.03 & -0.07 & -0.19 & 0.03 & 0.01 \\
-0.09 & 1.05 & -0.29 & 0.04 & 0.01 & -0.08 & -0.02 & -0.12 & 0.02 & -0.20 \\
0.04 & -0.07 & 1.03 & 0.03 & -0.00 & 0.04 & -0.20 & -0.09 & 0.03 & 0.01 \\
0.10 & -0.05 & 0.06 & 1.08 & -0.11 & 0.08 & -0.30 & -0.42 & 0.07 & 0.01 \\
-0.20 & 0.04 & -0.09 & -0.09 & 1.01 & 0.00 & -0.03 & -0.01 & 0.01 & -0.00 \\
0.04 & 0.01 & 0.01 & 0.03 & -0.10 & 1.02 & -0.10 & -0.10 & 0.02 & -0.00 \\
-0.10 & -0.08 & 0.04 & -0.10 & 0.02 & -0.09 & 1.06 & -0.01 & -0.10 & 0.01 \\
-0.10 & -0.07 & -0.07 & -0.11 & 0.02 & -0.10 & -0.02 & 1.11 & -0.10 & 0.01 \\
0.02 & 0.02 & 0.00 & 0.02 & -0.00 & 0.02 & -0.10 & -0.10 & 1.02 & -0.00 \\
0.01 & 0.02 & 0.01 & -0.08 & 0.01 & 0.01 & 0.04 & -0.17 & 0.01 & 0.99 \\
\hline
\end{tabular}

Source: Table I.

TABLE III Integer proportions of feasibility bounds for selected furniture distribution, $w_{10}=1$

\begin{tabular}{rlrrrrr}
\hline & & \multicolumn{2}{c}{ Upper bound } & & \multicolumn{2}{c}{ Lower bound } \\
& & $\mathbf{w}$ & $\mathbf{k}$ & & $\mathbf{w}$ & $\mathbf{k}$ \\
\hline 1 & Bench & 11 & 10.317 & & 1 & 0.645 \\
2 & Planter & 6 & 4.417 & & 2 & 1.324 \\
3 & Litter bin & 1 & 0.105 & & 1 & 0.707 \\
4 & Bollard & 2 & 0.002 & & 1 & 0.342 \\
5 & Railing & 11 & 8.698 & & 2 & 1.662 \\
6 & Gateway & 2 & 0.902 & & 1 & 0.729 \\
7 & Lighting & 4 & 2.257 & & 1 & 0.558 \\
8 & Signage & 2 & 0.023 & & 1 & 0.479 \\
9 & Canopy & 2 & 1.772 & & 1 & 0.896 \\
10 & Fountain & 1 & 0.995 & & 1 & 0.869 \\
\hline
\end{tabular}

change as a result of possible configuration change in street furniture throughout the streetscape.

Table I, representing the matrix $\mathbf{A}$, can be inverted in order to assess a possible distribution $\mathbf{k}$ of real items from a target distribution $\mathbf{w}$ of virtual items. Table II shows the values for $\mathbf{A}^{-1}$. Based on these values, and assuming that $\mathbf{A}$ remains constant regardless of possible variation in configuration, a target distribution $\mathbf{w}$ could be feasible, i.e. it could yield a nonnegative distribution $\mathbf{k}$ of visual, real items. Two vectors $\mathbf{w}$, representing approximate feasibility bounds (or their multiples) beyond which integer values $\mathbf{w}$ would be infeasible (i.e., $\mathbf{w A}^{-1}$ would yield negative values for $\mathbf{k}$ ) are presented in Table III. The matrix A as given by Table I is close to being a monotonous linear operator, and the values of $\mathbf{w}$, along with the corresponding values of $\mathbf{k}$, are the approximate extremes of the two corresponding feasibility ranges, with the category $w_{i}$ normalized to 1 for $i=10$.
From Eq. (4) it follows further that if $\mathbf{A}^{-1}$ is monotonous (or close to monotonous) then the higher is the value of the scalar $s, s \geq r(\mathbf{D})$, the larger also is the range of feasible solutions to

$$
\mathbf{w} \cdot \mathbf{A}^{-1}=\mathbf{k} \cdot(s \mathbf{I}-\mathbf{D}),
$$

where, as before,

$$
\mathbf{D} \geq \mathbf{0}, \quad s \geq r(\mathbf{D})>\mathbf{0}, \quad \mathbf{w}>\mathbf{0} .
$$

In such a case, however, the values of $\mathbf{w}$ and $\mathbf{k}$ also converge. Since A in Eq. (2) is a normalized matrix with its diagonal elements always 1 , the meaning of Eqs. (4) and (5) is that the feasibility range for Eq. (2) increases as positive offdiagonal elements of A decrease. This corresponds to the observation of Tables I-III.

\section{POST-MODERNIST URBAN DESIGN: UTILITY AS AN ORNAMENT}

The ambiguity $a_{i i}$ is always 1 by convention; but the lower the ambiguity $a_{i j}$ is, and the closer it is to zero, the less choice there is to use an item $i$ for an item $j$. Where such a choice decreases, any virtual distribution $\mathbf{w}$ is numerically close to a physical distribution $\mathbf{k}$, by definition of the two distributions. The more distinct the two distributions are from one another, the smaller is the feasibility range in Eq. (5). The challenge in the design (or the spatial configuration) of artificial street objects is in the measure of deliberate ambiguity that will imply a desirable virtual distribution, w, under an optimal, physical distribution, $\mathbf{k}$. 
The formal exposition of ambiguity involves the full domain of $n$ possible uses within a delineated environment. This appears to have two significant implications for contemporary urban design. First, a fundamental distinction between street furniture and most other forms of applied art must be made. While applied art is by and large static, the design of street furniture must take into consideration the physical movement of pedestrians. The Vitruvian comparison of the street to a theatre scene (Moughtin, 1991) is fundamental. The physical texture and appearance of streets has been changing over the ages (Krier, 1979), but their pedestrian use less so. If we accept the notion that walking, standing and sitting are perhaps the most primitive forms of a performing art, the street, with the pedestrians in it, must be perceived in a context different from that of most other applied art (cf. Olsen, 1986). Consideration of streetscapes that respects the variety among pedestrians, must also recognize their often deliberately ambiguous usage of street furniture. Street design thus becomes more analogous to a stage-setting in a performing art, than a static architectural construct.

The additional implication of ambiguity for urban design is in the specific category of use, "ornamentation." Within the scope of ambiguity introduced here, the notion of visual versus virtual items has a particularly interesting expression in post-modernist architecture. Frank Gehry's maverick design of his own home, deliberately exhibiting corrugated metals, plywood and chain-link fencing, is an excellent example. A utility item of a declared manufacturing purpose, such as plywood or industrial railing, emerges to fulfil the role of architectural ornamentation. Even an earlier, less explicit, archetype is Rob Krier's design concept of the Open Market Halls in Karlsruhe, Germany, which deliberately exposes supporting roof structures (Krier, 1982). And perhaps the earliest example, tracing the ambiguity notion between industrial utility and ornament back to the Art Nouveau era, is Antoní Gaudí's Sagrada Familia. Now the trade-mark of Barcelona's skyline, the construction cranes of Gaudís "Unfinished Cathedral" are the perpetual statement of artistic ambiguity nourished by 20 th century urban ambience (Akkerman, 1998). The very origins of the expressionist approach to architectural design can be possibly traced to Philip Speakman Webb (1831-1915), known for having designed furniture for William Morris, but also for his intentional exposure of materials in his designs and for showing pieces of working equipment as parts of buildings.

The possibility for an item to serve as a surrogate for another item is a challenge that has been always addressed by designers (de Lucio-Meyer, 1973). Examples of ambiguous furniture design, in particular, are Jere Osgood's (1986) “Writing Desk," or Wendell Castle's (1987) "Time to Say Goodbye Clock." Some examples of ambiguous design of street items are sculptor Siah Armajani's pedestrian bridge in Minneapolis, which serves as a wonderful piece of public art, or Scott Burton's granite sculptures - benches overlooking New York Harbour from Battery Park.

Could deliberate use of street hardware as an embellishing feature in streetscape design be the next step? The placement of municipal hardware in back alleys, or in other shunned areas of the NorthAmerican downtown, clearly perpetuates the derogation of these precincts as criminogenic agents. Pedestrianization strategies for urban centers so far, however, have missed addressing the potential of street hardware as an opportunity to enhance, rather than to avoid, its immediate physical environment. The satirizing of exposed pipes and other conveyor utilities as explicit streetscape features, would introduce a rustic element into the formal city scene. By offering a unique opportunity for playful, ambiguous use, obsolete municipal fixtures could turn some downtown areas into pedestrian routes frequented by children and adults alike.

\section{CONCLUSION}

The ambiguity matrix A maps the potential of declared items in urban space, to serve as surrogates for other items. The closer $\mathbf{A}$ is to the identity matrix $\mathbf{I}$, 
the more resemblance there is between a desired set of functions in the environment, and an actual distribution of items: items formally declared fulfil their so-declared function and no other, without ambiguity. On the other hand, with $\mathbf{A}$ attaining positive offdiagonal values, ambiguity is deliberately introduced into the environment indicating informal usage to the declared items.

From the perspective of usage, deliberate ambiguity is most interesting within the narrow range of variation of numeric values in the vector $\mathbf{w}$, which allow for feasible vectors k. From Eq. (5) it follows that $\mathbf{A}$, as a measure of mutual ambiguity (and availability) between items, is most interesting with the largest possible offdiagonal entries. Within this paradigm the notion of ambiguity is quite literally analogous to the notion of connotation used both in linguistics and in the semiotics of objects (Krampen, 1979). In a sense, the distinction between virtual usage and declared usage of items can be seen analogous to the notion of slang as distinct from the lexical mode of spoken or written language. And pursuant to this analogy, the ambiguity notion can be further compared to humor. The city street, which has always been the source of new informal expressions in the spoken language, should also be a source of opportunity for an informal physical expression and visual pun. Within the notion of spatial ambiguity, the choreographic approach to streetscape as well as the instrumentalist approach that follows Gehry, both seem to offer an opportunity to redefine the characteristics, and the numbers, of the pedestrian population in our streets.

In spite, and perhaps because, of the difference between architecture and street design, the cited examples of deliberate ambiguity in applied art may herald the emergence of a post-modernist urban design, precisely within the image of Gehry's unpretentious architectural expressionism. This notion applied to street furniture leads, in particular, to a call to utilize the back alleys of NorthAmerican downtown areas as pedestrian routes rusticated by deliberately ambiguous use in street hardware. At the time of often superficial cries for sustainable urban development, nothing could elevate better a suppressed urban truth into the image of a rediscovered beauty.

\section{References}

Akkerman, A. (1992) Fuzzy targeting of population niches in urban planning and the fractal dimension of demographic change. Urban Studies 29, 1093-1114.

Akkerman, A. (1998) Place and Thought: The Built Environment in Early European Philosophy. London, Woodridge, pp. 154 170.

Broadbent, G. (1990) Design in Architecture: Architecture and the Human Sciencs. London, David Fulton, pp. 338-344.

Berman, A. and Plemmons, R.J. (1979) Nonnegative Matrices in the Mathematical Sciences. New York, Academic Press, pp. $132-164,270-291$.

de Lucio-Meyer, J.J. (1973) Visual Aesthetics. London, Harper \& Row Publishers.

Gibbons, J. and Oberholzer, B. (1991) Urban Streetscapes: A Workbook for Designers. London, BSP Professional Books.

Kim, T.J. (1992) Expert systems in urban planning: Future research directions, in T.J. Kim, L.L. Wiggins and J.R. Wright (Eds.) Expert Systems: Applications to Urban Planning. Berlin, Springer-Verlag, pp. 223-224.

Krampen, M. (1979) Meaning in the Urban Environment. London, Pion, pp. 18-27.

Kreir, R. (1982) On Architecture. London, Academy Editions, and New York, St. Martin's Press, p. 88.

Krier, R. (1979) Urban Space. New York, Rizzoli, p. 17.

Moughtin, C. (1991) The European city street, Part 1: Paths and places. Town Planning Review 62, 51-77.

Moughtin, C. (1992) Urban Design: Street and Square. London, Butterworth-Heinemann, pp. 127-128.

Olsen, D.J. (1986) The City as a Work of Art. New Haven and London: Yale University Press, pp. 37-38.

Pennartz, P.J.J. (1992) The symbolic infrastructure of an environmental art project. Environment and Planning B: Planning and Design 19, 205-220.

Sainsbury, M. (1994) Why the world cannot be vague, The Southern Journal of Philosophy 33(Suppl.), 63-81.

Scott, C. (1993) The semiotics of retail space: An application of the repertory grid methodology, Semiotica 94, 295-303.

Venturi, R., Scott-Brown, D. and Izenour, S. (1972) Learning from Las Vegas. MIT Press, Cambridge, Mass, and London, England, p. 71. 


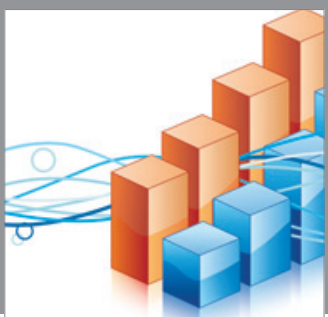

Advances in

Operations Research

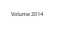

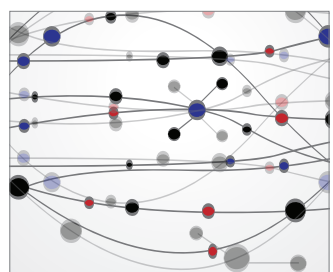

\section{The Scientific} World Journal
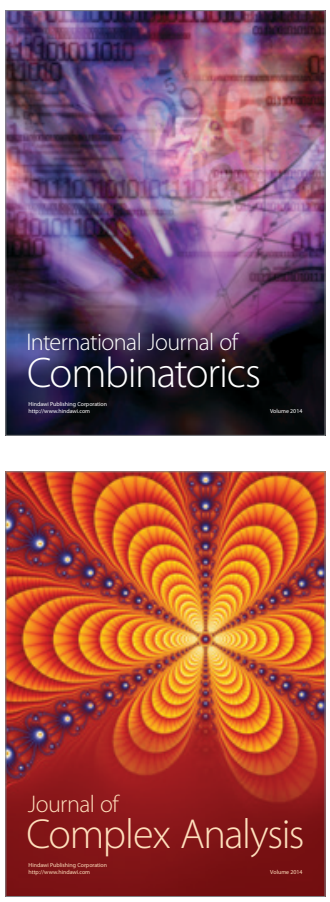

International Journal of

Mathematics and

Mathematical

Sciences
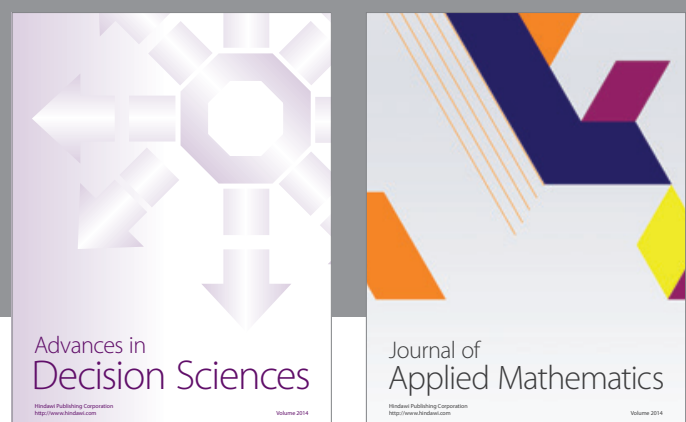

Journal of

Applied Mathematics
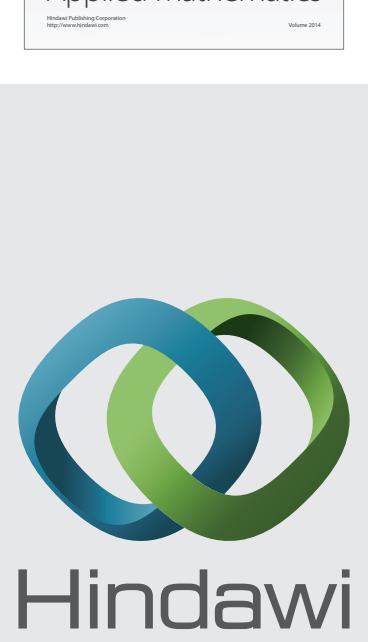

Submit your manuscripts at http://www.hindawi.com
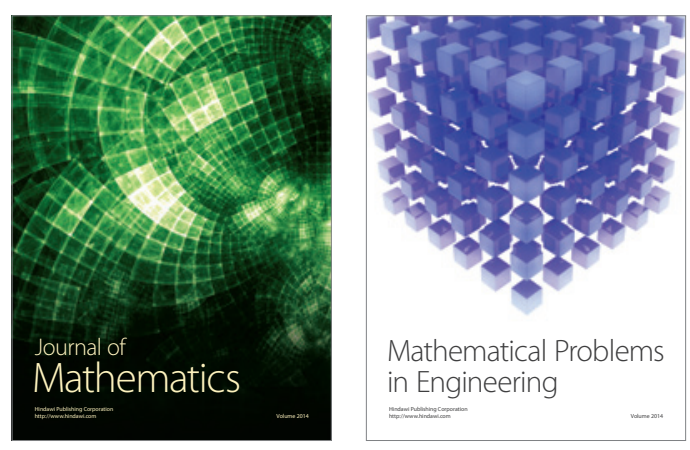

Mathematical Problems in Engineering
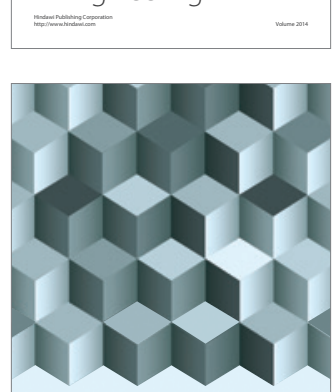

Journal of

Function Spaces
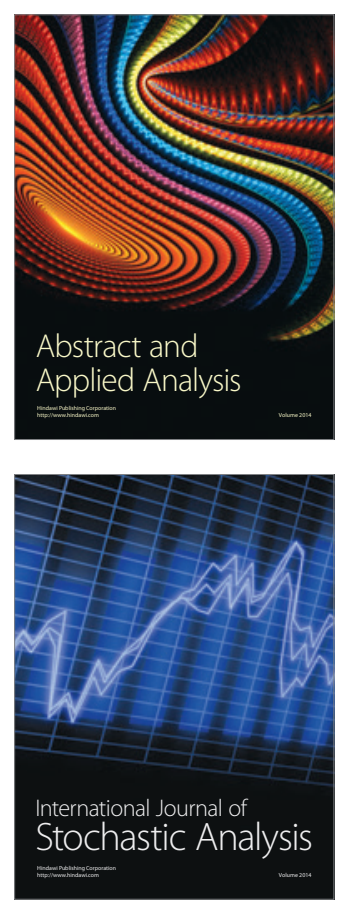

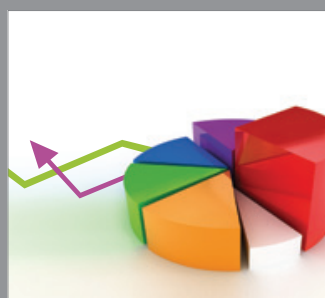

ournal of

Probability and Statistics

Promensencen
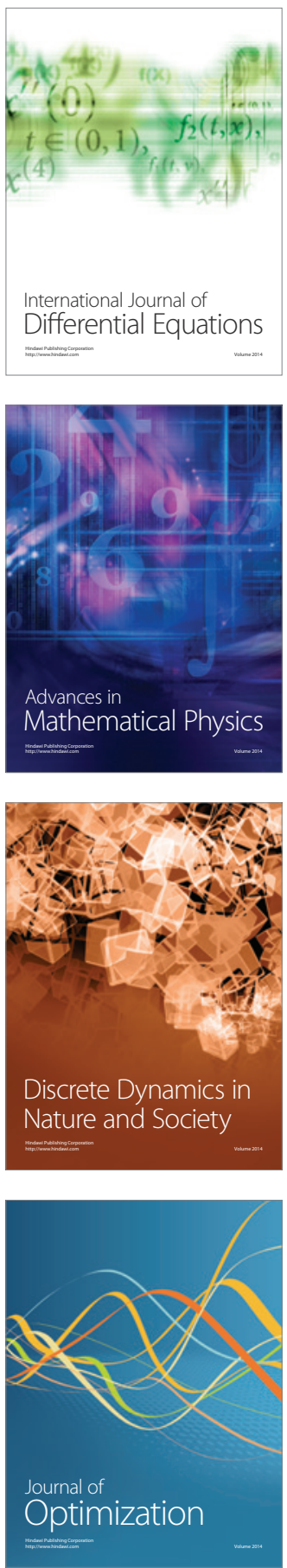